\title{
CAPACIDAD DE CARGA TURÍSTICA, BASE PARA EL MANEJO SUSTENTABLE: GRUTA DE HUAGAPO
}

\author{
TOURIST CARRYING CACACITY, BASIS FOR SUSTAINABLE \\ MANAGEMENT: HUAGAPO GRO'T'TO \\ (Di) Huaroc Ponce Edwin Jhon ${ }^{*}$, (D) Raqui Ramirez Christian Efrain ${ }^{1}$, (DD Jurado Taipe Ralph \\ Sandy ${ }^{1}$, (D) Huaroc Ponce Nilton Marx ${ }^{2}$ \\ ehuaroc@uncp.edu.pe; craqui@uncp.edu.pe; raphomania@hotmail.com; nhuaroc@uniscjsa.edu.pe \\ ${ }^{1}$ Universidad Nacional del Centro del Perú, Junín, Perú \\ ${ }^{2}$ Universidad Daniel Alcides Carrión, Lima, Perú \\ *Correspondencia: Huaroc Ponce Edwin Jhon. Email: ehuaroc@uncp.edu.pe
}

Recibido: 13.08.2020 | Aprobado: 10.10.2020

\section{RESUMEN}

La gruta de Huagapo ubicada en el distrito de Palcamayo, provincia de Tarma, región Junín - Perú, a 3572 m.s.n.m. Fue recorrida hasta los 2800 metros de profundidad, para el uso turístico se recorre 212 metros (ruta seca). La investigación se basa en el análisis de la capacidad de carga turística, con la metodología de M. Cifuentes, hallando la capacidad de carga física CCF, los factores de corrección Fc, la capacidad de carga real CCR, la capacidad de manejo CM, y la capacidad de carga efectiva CCE, o llamada capacidad de carga turística CCT. Los resultados fueron: $\mathrm{CCF}=2692$ visitas; $\mathrm{Fc}=$ $0.324002 ; \mathrm{CCE}=872$ visitantes y la capacidad de carga turística resulto 483 visitantes al día.

Palabras clave: Ecología, sostenibilidad y capacidad de carga turística.

\section{ABSTRACT}

Huagapo grotto is located in Palcamayo district Tarma province, Junín region Peru, at 3572 m.s.n.m. It was crossed until 2800 meters of depth, for the tourist use it is crossed 212 meters (dry route). The investigation is based on the analysis of the tourist carrying capacity, with Cifuentes's methodology, finding the physical carrying capacity $\mathrm{CCF}$, the correction factors Fc, the real carrying capacity CCR, the management capacity $\mathrm{CM}$, and the effective carrying capacity CCE, or called tourist carrying capacity CCT. The results were: $\mathrm{CCF}=2692$ visits; $\mathrm{Fc}=0.324002 ; \mathrm{CCE}=872$ visitors and the tourist carrying capacity resulted in 483 visitors per day.

Keywords: Ecology, sustainability and tourist carrying capacity. 


\section{INTRODUCCIÓN}

A nivel mundial hoy en día el turismo se ha convertido en una actividad económica importante para el desarrollo de la población (Laurente \& Machaca, 2020), la movilización de las personas es promovido por viajes más accesibles, tecnología al alcance de todos, mejora en la economía de la clase media, negocios innovadores en el sector y entrega de visas en menor tiempo; el turismo internacional creció un $5 \%$ el 2018 con mil cuatrocientos millones de turistas y con 1,7 billones de dólares americanos referente a los ingresos de exportación (UNWTO, 2019), este comportamiento no es solo ahora, se presentó en años anteriores (UNWTO, 2018).

La actividad turística debe ir de la mano con el cuidado del medio ambiente no solo por las empresas y los destinos visitados (Cornejo, 2019; Cardoso, 2006), este cambio de patrones en el turismo está presente en la demanda (Perruolo \& Camargo, 2017; Yang, 2017), ya que el modelo convencional pierde vigencia en la actualidad (Caicedo et al., 2018; Sati, 2018a; Guzmán-López et al., 2011; Simancas, 2006), dando paso al turismo sostenible como modelo para su ejecución (Cornejo et al., 2019; Puente et al., 2011; López \& López, 2008).

La Capacidad de Carga Turística es una herramienta que ayuda a la planificación de los espacios naturales (Fernández \& Lazovski, 2018; Montalvo \& Castillo, 2018; Zhang et al., 2017; Dias et al., 2012; Del Valle, 2011; Varisto et al., 2009; Cifuentes, 1999), la CCT puede variar según el lugar y el año, es indispensable un monitoreo como proceso gradual para su correcta planificación, manejo e investigación de los recursos turísticos (SoriaDíaz \& Soria-Solano, 2015; Amador et al.,1996), la CCT junto a los estudios de impacto ambiental son metodologías que evitan el uso excesivo, degradación y depredación de lugares de interés (Varisto et al., 2009; López \& López,2008).

\section{REVISIÓN DE LITERATURA}

El desarrollo sostenible del turismo es mencionado en la Cumbre de Río de Janeiro donde se propone la Agenda 21 para la preservación de los recursos naturales mediante modelos de desarrollo (ONU, 1992), posterior a ello se crea la Carta del Turismo Sostenible en Lanzarote, donde se menciona que el desarrollo turístico debe ser a largo plazo con beneficio directo a la población que circunda el atractivo (ONU et al., 1995). En el evento "El Futuro que queremos" se expuso acerca de los objetivos del desarrollo sostenible y la economía verde para la economía de los pueblos (ONU, 2012), el 2015 en la Carta Mundial de Turismo Sostenible en Vitoria-Gasteiz se analizó 03 de los 17 objetivos para el desarrollo sostenible de la Organización de las Naciones Unidas (crecimiento económico, crecimiento y producción sostenible y conservación de los recursos), también se discutió sobre países con escazas políticas de turismo sostenible y la educación para el desarrollo vinculado al turismo (Consejo Global de Turismo Sostenible \& Instituto de Turismo Responsable, 2015). En la actualidad los destinos responsables con el medio ambiente es uno de los objetivos de la Agenda 2030, denominado Convención Marco sobre el Cambio Climático y el Pacto Mundial de los alcaldes por el clima y la Energía (Espinoza et al., 2017).

El turismo sostenible se refiere al cuidado actual y futuro en lo social, económico y medioambiental para satisfacer las necesidades de los actores de la actividad turística 
(Blancas et al., 2010), es preponderante establecer límites para alargar la vida del recurso para las siguientes generaciones (Segrado et al., 2017; Simancas, 2006), la CCT tiene relación con el turismo sostenible ya que buscan el cuidado del medio natural (Mc Coy, 2016; Ibañez, 2016; Marozzi et al., 2011; Vera et al., 2011; López \& López, 2008).

La capacidad de carga es un término acuñado a la ecología y es la capacidad máxima de un grupo de personas en un determinado momento (Gutierrez-Fernández \& Sierra, 2015; Hui, 2006), este término se unificó con el turismo y es el número máximo de personas que puede albergar un recurso turístico para el goce de los visitantes, la calidad y la preservación del lugar (Cornejo et al., 2019; Mestanza et al., 2019; Crispin et al., 2017; Tudela \& Giménez, 2008; Rosell \& Del Pozo, 2007; García, 2003; Wearing \& Neil, 1999; Amador et al., 1996).

En la actualidad no hay una definición concreta, una metodología y beneficios a futuro de la CCT (Matos \& Pérez, 2019; López \& López, 2008; Saveriades, 2000), este tema pasó de ser tratado de manera unidimensional a ser integral donde la parte física ahora se complementa con lo social, ambiental, económico y político (Alvarado et al., 2018; Salerno et al., 2013; García et al., 2011).

La gruta de Huagapo fue recorrida hasta los 2800 metros (Huari \& Salazar, 2006), al ingreso hay figuras rupestres tales como zoomorfas, cuerpos celestes y figuras antropomorfas que datan de 6000 a.c. (Castro, 2000), se hizo varias expediciones, en 1969 dirigida por Jose C. Bein hasta los 1000 metros de profundidad, en 1972 la expedición británica Imperial College exploró hasta los 1600 metros, en 1976 la expedición francesa Marbre Agir hasta los 1618 metros, en 1988 la expedición compuesta por el Centro de exploraciones subterráneas del Perú (CEESPE) y la Federación de Caza Submarina del Perú quienes llegaron a los 2000 metros y en 1994 hasta los 2200 metros de profundidad y una expedición franco-peruana exploraron hasta los $2800 \mathrm{~m}$ (El Comercio, 2007), actualmente para el turismo se hace uso de la ruta seca con 212 metros de profundidad.

\section{Figura 1}

Perfil de la gruta de Huagapo.

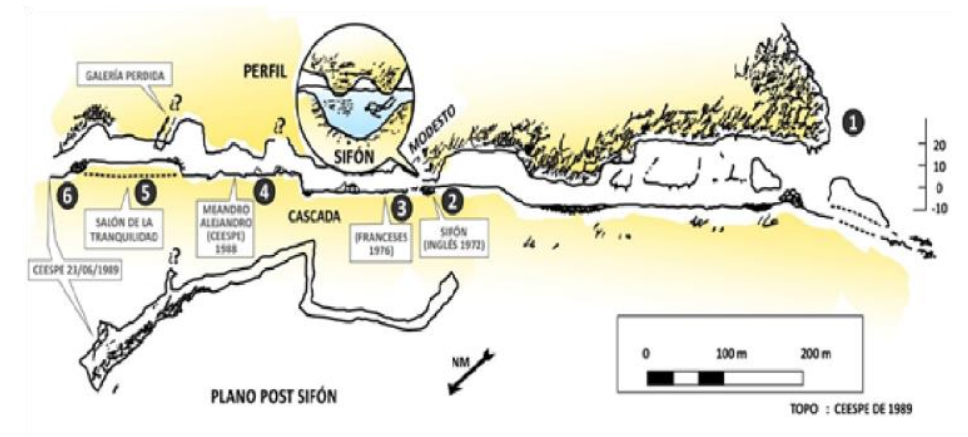

Fuente: Elaboración con base a la Publicación El Comercio (2007).

Nota. 1. Entrada, 2. sifón, 3. punto donde llegó la expedición francesa, 4. punto donde llegó la expedición CEESPE (1988), 5. salón de la tranquilidad, 6. punto donde llegó la expedición CEESPE (1989). 


\section{MATERIALES Y MÉTODOS}

Se usó la metodología de Miguel Cifuentes Arias para hallar la CCT, que mide los factores físicos e indicadores de manejo (Cifuentes, 1999).

\section{Capacidad de carga Física}

Cálculo CCF

\begin{tabular}{|c|c|c|c|}
\hline & $C C F=\left(\frac{S}{S p}\right) \times N V$ & & $N V=\frac{H v}{T v}$ \\
\hline$S^{57}$ & $\begin{array}{l}\text { Superficie disponible. Es la longitud de del } \\
\text { Sendero (m) } 0 \text {, en áreas abiertas, el área } \\
\text { disponible }\left(\mathrm{m}^{2}\right)\end{array}$ & $H v^{59}$ & Horario de visitas \\
\hline Sp & $\begin{array}{l}\text { Superficie utilizada por una persona }{ }^{50} \text { Para } \\
\text { senderos el Sp se define como la distancia } \\
\text { ocupada por una persona (1m) más la distancia } \\
\text { mínima entre personas ( } 1 \mathrm{~m}) \text {. Para áreas abiertas } \\
\text { se define el espacio mínimo como } 1 \mathrm{~m}^{2}\end{array}$ & $T v^{60}$ & $\begin{array}{l}\text { Tiempo necesario para visitar } \\
\text { cada sitio }\end{array}$ \\
\hline NV/día & $\begin{array}{l}\text { Número de veces que el sitio puede ser visitado } \\
\text { por una persona en un día }\end{array}$ & & \\
\hline
\end{tabular}

Fuente: Cifuentes

\section{Capacidad de carga Real}

Cálculo CCR

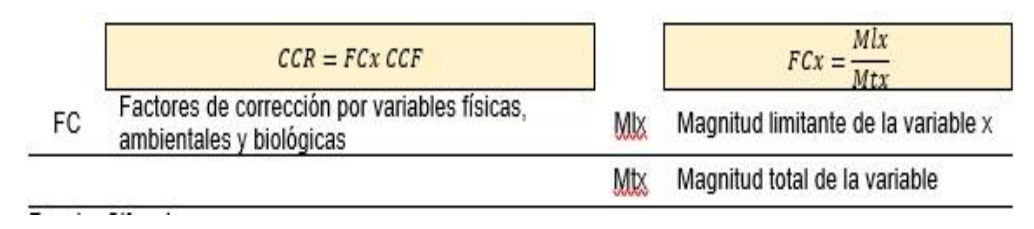

Fuente: Cifuentes

\section{Tabla 1}

Niveles de riesgo de erosión de pendiente y niveles de dificultad.

\begin{tabular}{lll}
\hline Pendiente & Grado de erodabilidad $>$ & Factor de ponderación \\
\hline$>=10 \%$ & Bajo & 0 \\
$10 \%-20 \%$ & Medio & 1 \\
$>=20 \%$ & Alto & 1.5 \\
\hline
\end{tabular}

Fuente: Cifuentes

\section{Tabla 2}

Valores para calificación de criterios (grados, cantidad, estado, localización y funcionalidad)

\begin{tabular}{lll}
\hline Calificación & Valor & Porcentaje (ponderación) \\
\hline Insatisfactorio & 0 & $\leq 35 \%$ \\
Poco satisfactorio & 1 & $36 \%-50 \%$ \\
Medianamente satisfactorio & 2 & $51 \%-75 \%$ \\
Satisfactorio & 3 & $76 \%-89 \%$ \\
Muy satisfactorio & 4 & $\geq 90 \%$ \\
\hline
\end{tabular}

Fuente: Cifuentes 


\section{Capacidad de Manejo}

\section{Cálculo CM}

$$
C M=\frac{\text { instalaciones turísticas }+ \text { equipamiento }+ \text { personal }}{3} \times 100
$$

Fuente: Cifuentes

\section{Capacidad de carga Efectiva:}

Cálculo de CCE

\begin{tabular}{ll}
\hline & CCE $=$ CCR $\times$ CM \\
\hline CCR & Capacidad de Carga Real \\
CM & Capacidad de manejo \\
\hline
\end{tabular}

Fuente: Cifuentes

\section{RESULTADOS}

Para calcular la CCT se tomaron en consideración los siguientes supuestos: i) Horario de visita, 10 horas diarias de 8:00 a 18:00 horas ii) Tiempo estimado del recorrido, noventa minutos, iii) Longitud del sendero es 525 metros, iv) Funcionamiento todo el año

Cálculo de la Capacidad de Carga Física (CCF) Fue estimado mediante la siguiente expresión matemática:

$$
C C F=\left(\frac{S}{S p}\right) \times N V
$$

$\mathrm{S}=525$ metros lineales.

$\mathrm{Sp}=1.50$ metros

$\mathrm{NV} /$ día $=7.69$

Para hallar el cálculo de (NV) se requirió el número de veces que la gruta puede ser visitada en un día, el cual fue hallado con el cálculo:

$$
N V=\frac{H v}{T v}
$$

$\mathrm{Hv}=10$ horas de atención al público

$\mathrm{Tv}=1.30$ horas (estimado para el recorrido)

$\mathrm{NV}=7.69$

Se obtuvo la CCF de 2691.50 visitas/día.

Cálculo de la CCR

Es considerado el límite real de visitas al atractivo turístico el cual se debe determinar a partir de la CCF, sometida a factores de corrección previamente definidos teniendo en cuenta las particularidades del atractivo. Dichos factores pueden ser: variables físicas, ambientales, biológicos y de manejo administrativo, se expresa con la ecuación:

$$
C C R=F C x C C F
$$


Los factores de corrección son:

Factores sociales: Mide los aspectos referidos a la operación del servicio, como el manejo de grupos en visita y la distancia entre estos grupos, asegurando con esto una calidad adecuada de visita y disfrute de los visitantes, cuya fórmula es:

$$
F C x=\frac{M l x}{M t x}
$$

Obteniendo los siguientes resultados:

$\mathrm{Ml}=2946.85$ metros

$\mathrm{Mt}=5378$ metros

\begin{tabular}{|c|c|c|c|c|}
\hline $\mathrm{Ml}=\mathrm{AT}-\mathrm{AO}$ & $---->$ & $\mathrm{AT}=$ Área total & $---->$ & $\mathrm{AO}=$ Área ocupada \\
\hline $\mathrm{AO}=\mathrm{P} \times \mathrm{Dp}$ & $---->$ & $\begin{array}{l}\mathrm{P}=\text { Personas simultaneas } \\
\text { en el recorrido }\end{array}$ & $--->>$ & $\begin{array}{l}\text { Dp }=\text { Distancia por } \\
\text { persona }\end{array}$ \\
\hline $\mathrm{P}=\mathrm{NG} \times \mathrm{pg}$ & $---->$ & $\mathrm{NG}=$ número de grupos & $--->$ & $\begin{array}{l}\mathrm{pg}=\text { personas por } \\
\text { grupo }\end{array}$ \\
\hline $\mathrm{NG}=\mathrm{AT} /(\mathrm{pg} \times \mathrm{dp})+\mathrm{dtg})$ & $---->$ & $\mathrm{AT}=$ área total & $---->$ & $\begin{array}{l}\mathrm{pg}=\text { personas por } \\
\text { grupo }\end{array}$ \\
\hline & $---->$ & \multirow{2}{*}{\multicolumn{3}{|c|}{$\mathrm{Dp}=$ área que ocupa una persona en el grupo }} \\
\hline & $---->$ & & & \\
\hline
\end{tabular}

$\mathrm{AT}=5378 \mathrm{~m}$. cuadrados

$\mathrm{AO}=2431.149$ pax

$\mathrm{P}=1620.766$ personas

$\mathrm{Dp}=1.5 \mathrm{~m}$

$\mathrm{NG}=147.342 \mathrm{Pg}=11$ pax dp $=1.5 \mathrm{~m} \mathrm{dtg}=20 \mathrm{~m}$

El resultado del FCsoc integró diferentes factores como la distancia entre grupos, número de personas por grupo y el espacio mínimo utilizado por el individuo en la visita a la gruta, el resultado fue 0.547945 .

Factores Físicos: Están relacionados con la superficie, la erosión y la accesibilidad, considerando:

Factor de corrección de erodabilidad (FCero): Se medió la susceptibilidad del suelo, a la erosión por diferentes acciones (agua, viento, pendiente, tipo de suelo, vegetación como cobertura y manejo de la tierra a raíz de la visita), con la siguiente ecuación:

$$
\text { FCero }=1-\frac{\max 1.5+m m x 1}{m t}
$$

$\mathrm{ma}=247 \mathrm{~m}$. lineales $\mathrm{mm}=212 \mathrm{~m}$. lineales $\mathrm{mt}=525 \mathrm{~m}$. lineales FCero $=-0.010952$

El resultado de este factor limita el número de visitas en el sendero, considerando la posibilidad de erosión, el cual fue -0.010952 . 
Factor de corrección de accesibilidad (FCacc): Se analizó el grado de la dificultad que tiene los visitantes al momento de desplazarse considerando la pendiente del sendero, se calculó con la siguiente fórmula:

$$
F C a c c=1-\frac{\max 1.5+m m \times 1}{m t}
$$

$\mathrm{Ma}=84$

$\mathrm{m}$. sendero $+126.60 \mathrm{~m}$. gruta $=210.60 \mathrm{~m} \mathrm{Mm}=112 \mathrm{~m}$. sendero $+85.40 \mathrm{~m}$. gruta $=$ $197.40 \mathrm{~m}$.

$\mathrm{Mt}=525 \mathrm{~m}$

FCacc $=0.02229$

El resultado de este factor limita el desplazamiento considerando la pendiente del sendero, el cual fue 0.02229 .

Factores ambientales: Considera las precipitaciones y el brillo solar como factores de corrección.

Factores de corrección de precipitación (FCprec): Se midió la susceptibilidad del suelo a la erosión a raíz de las precipitaciones con mayor frecuencia (desde la quincena de diciembre a la quincena de marzo), se usó la fórmula:

$$
\text { FCprec }=1-\frac{h l}{h t}
$$

$\mathrm{Hl}=5$ horas diarias $\mathrm{x} 120$ días $=600$ horas año

$\mathrm{Ht}=3650$ horas

FCprec $=0.83562$

El cual limita la visita al atractivo por precipitaciones (lluvias), cuyo resultado fue 0.83562 .

Factor de corrección por brillo solar (FCbrill): Se midió el mayor grado de brillo solar el cual impide el disfrute de la gruta, el cual se percibe desde las 10 hasta las 15 horas del día, utilizando la siguiente fórmula:

$$
\text { FCbrill }=1-\left(\frac{h s o l}{h t}\right) \times \frac{m s}{m t}
$$

hsol $=980$ horas $\mathrm{ht}=3650$ horas $\mathrm{ms}=313$ metros $\mathrm{mt}=525$ metros FCbrill $=0.83993$

Limita las actividades por excesivo brillo solar. Teniendo un resultado 0.83993.

Factores de visita: Son el cierre temporal y el anegamiento.

Factor de cierres temporales (FCtem): Se analizó el impacto que genera el cierre temporal, prohibiendo las visitas, se usó la siguiente fórmula: 


\section{FCtem $=1-\frac{h c}{h t}$}

hc $=0$ horas ht $=3650$ horas

FCtem $=1$

Este factor limita la visita por mantenimiento, cuyo resultado es 1.

Factor de corrección de anegamiento (FCane): Se utilizó para los sectores donde el agua se estanca y las pisadas en el área tiende a dañar el recorrido, se usó la siguiente fórmula:

$$
\text { FCane }=1-\frac{m a}{m t}
$$

$\mathrm{Ma}=82.70 \mathrm{~m}$

$\mathrm{Mt}=525 \mathrm{~m}$

FCane $=0.424348$

El cual limita la visita por estancamiento de agua en diferentes sectores, cuyo resultado fue 0.424348 .

\section{Cálculo de la Capacidad de Manejo (CM)}

Se analiza la administración del atractivo con las siguientes variables: instalaciones, equipamiento (cantidad, estado, localización y funcionalidad) y en personal (cantidad, conocimiento y servicio al cliente), se usó la siguiente fórmula:

$$
C M=\frac{\text { instalaciones turísticas }+ \text { equipamiento }+ \text { personal }}{3} \times 100
$$

Tabla 3

\begin{tabular}{|c|c|c|c|c|c|c|c|c|}
\hline \multirow{2}{*}{$\mathbf{N}^{\circ}$} & \multirow{2}{*}{$\begin{array}{l}\text { Instalación } \\
\text { turística }\end{array}$} & \multirow{2}{*}{$\begin{array}{l}\text { Cantidad } \\
\text { actual }\end{array}$} & \multicolumn{4}{|c|}{ FACTOR } & \multirow{2}{*}{$\begin{array}{l}\text { Suma de } \\
\text { factores } \\
(\mathbf{S})\end{array}$} & \multirow{2}{*}{$\begin{array}{l}\text { Factor } \\
\text { Inter.= } \\
\left(4 x N^{\circ} \text { Fact. }\right)\end{array}$} \\
\hline & & & Cantidad & Estado & Localización & Función & & \\
\hline 1 & Estacionamiento & 1 & 3 & 3 & 3 & 3 & 12 & 0.6 \\
\hline 2 & Área de artesanía & 1 & 3 & 3 & 3 & 3 & 12 & 0.6 \\
\hline 3 & Área de Tópico & 1 & 1 & 1 & 1 & 1 & 4 & 0.2 \\
\hline 4 & $\begin{array}{l}\text { Servicios } \\
\text { higiénicos }\end{array}$ & 1 & 2 & 3 & 2 & 3 & 10 & 0.5 \\
\hline 5 & Sendero & 1 & 4 & 4 & 4 & 4 & 16 & 0.8 \\
\hline 6 & $\begin{array}{l}\text { Zona de } \\
\text { recreación }\end{array}$ & 2 & 4 & 4 & 4 & 4 & 16 & 0.8 \\
\hline \multicolumn{8}{|c|}{ PROMEDIO } & 0.5833333 \\
\hline
\end{tabular}

Factores de corrección para el cálculo de CM (Infraestructura)

Fuente: Cifuentes

Nota: El cálculo del CM con respecto a infraestructura considerando estacionamiento, área artesanal, tópico, servicios higiénicos, sendero y zona de recreación tiene un factor de corrección de 0.5833333 . 


\section{Tabla 4}

Análisis de factores de corrección para el cálculo de CM (Equipamiento)

\begin{tabular}{|c|c|c|c|c|c|c|c|c|}
\hline \multirow{2}{*}{$\mathbf{N}^{\circ}$} & \multirow{2}{*}{$\begin{array}{l}\text { Instalación } \\
\text { turística }\end{array}$} & \multirow{2}{*}{$\begin{array}{l}\text { Cantidad } \\
\text { actual }\end{array}$} & \multicolumn{4}{|c|}{ FACTOR } & \multirow{2}{*}{$\begin{array}{l}\text { Suma de } \\
\text { factores } \\
\text { (S) }\end{array}$} & \multirow{2}{*}{$\begin{array}{l}\text { Factor } \\
\text { Inter. }= \\
\left(4 \times N^{\circ} \text { Fact. }\right)\end{array}$} \\
\hline & & & Cantidad & Estado & Localización & Función & & \\
\hline 1 & $\begin{array}{l}\text { Botiquín } \\
\text { (primeros } \\
\text { auxilios) }\end{array}$ & 1 & 1 & 1 & 1 & 1 & 4 & 0.2 \\
\hline 2 & Extinguidores & 1 & 1 & 1 & 1 & 1 & 4 & 0.2 \\
\hline 3 & $\begin{array}{l}\text { Teléfono } \\
\text { público }\end{array}$ & 0 & 1 & 1 & 1 & 1 & 4 & 0.2 \\
\hline 4 & $\begin{array}{l}\text { Vehículo } \\
\text { (para } \\
\text { emergen- } \\
\text { cias) }\end{array}$ & 0 & 1 & 1 & 1 & 1 & 4 & 0.2 \\
\hline 5 & $\begin{array}{l}\text { Ta ch o s } \\
\text { de basura }\end{array}$ & 6 & 3 & 3 & 3 & 3 & 12 & 0.6 \\
\hline 6 & $\begin{array}{l}\text { Mesas y } \\
\text { bancos }\end{array}$ & 5 & 4 & 4 & 4 & 4 & 16 & 0.8 \\
\hline 7 & Señalización & 1 & 4 & 4 & 4 & 4 & 16 & 0.8 \\
\hline PRC & OMEDIO & & & & & & & 0.4285714 \\
\hline
\end{tabular}

\section{Fuente: Cifuentes}

Nota: El cálculo del CM con respecto a equipamiento considerando las instalaciones turísticas como botiquín, extinguidores, teléfono público, vehículo para emergencias, tachos de basura, mesas y bancos y señalización tiene un factor de corrección de 0.4285714 .

\section{Tabla 5}

Análisis de factores de corrección para el cálculo de CM (Recursos Humanos)

\begin{tabular}{|c|c|c|c|c|c|c|c|}
\hline \multirow{2}{*}{$\mathbf{N}^{\circ}$} & \multirow{2}{*}{$\begin{array}{l}\text { Instalación } \\
\text { turística }\end{array}$} & \multirow{2}{*}{$\begin{array}{l}\text { Cantidad } \\
\text { actual }\end{array}$} & \multicolumn{3}{|c|}{ FACTOR } & \multirow{2}{*}{$\begin{array}{l}\text { Suma de } \\
\text { factores } \\
\text { (S) }\end{array}$} & \multirow{2}{*}{$\begin{array}{l}\text { Factor Inter. } \\
=\left(4 \times N^{\circ} \text { Fact. }\right)\end{array}$} \\
\hline & & & Cantidad & Conocimiento & $\begin{array}{l}\text { Atención } \\
\text { al cliente }\end{array}$ & & \\
\hline 1 & $\begin{array}{l}\text { Personal de } \\
\text { boletería }\end{array}$ & 1 & 3 & 3 & 3 & 9 & 0.6 \\
\hline 2 & Guías & 1 & 4 & 4 & 4 & 12 & 0.8 \\
\hline 3 & $\begin{array}{l}\text { Conductores de } \\
\text { vehículos públicos }\end{array}$ & 0 & 3 & 3 & 3 & 9 & 0.6 \\
\hline 4 & Fotógrafos & 1 & 3 & 3 & 3 & 9 & 0.6 \\
\hline PRC & OMEDIO & & & & & & 0.65 \\
\hline
\end{tabular}

Fuente: Cifuentes 
Nota: El cálculo del CM con respecto a recursos humanos considerando el personal de boletería, guías, conductores de vehículos públicos y fotógrafos tiene un factor de corrección de 0.65 .

$\mathrm{CM}=$ (Factor por instalaciones turísticas + Factor equipamiento + Factores de personal) $/ \mathrm{N}^{\circ}$ criterios

\begin{tabular}{ll}
\hline Infraestructura & 0.5833 \\
\hline Equipamiento & 0.4285 \\
Personal & 0.65 \\
\hline Total & 1.66 \\
\hline
\end{tabular}

Capacidad de manejo 55.39

La CM alcanzó un 55.4 \%, cabe precisar que la deficiencia más grande se concentró en el equipamiento.

Cálculo de la Capacidad de Carga Efectiva (CCE)

Una vez calculada la CM, se calculó la CCE de la siguiente manera:

$\mathrm{CCE}=\mathrm{CCR} * \mathrm{CM}$. Entonces: $\mathrm{CCE}=872$ visitas $/$ día $* 55.39 \% \mathrm{CCE}=483$ visitas $/$ día.

El número máximo de visitas que se debe permitir en la gruta de Huagapo considerando las características físicas y su capacidad de manejo es de 483 visitantes al día.

\section{Figura 2}

Zonas de distribución del atractivo

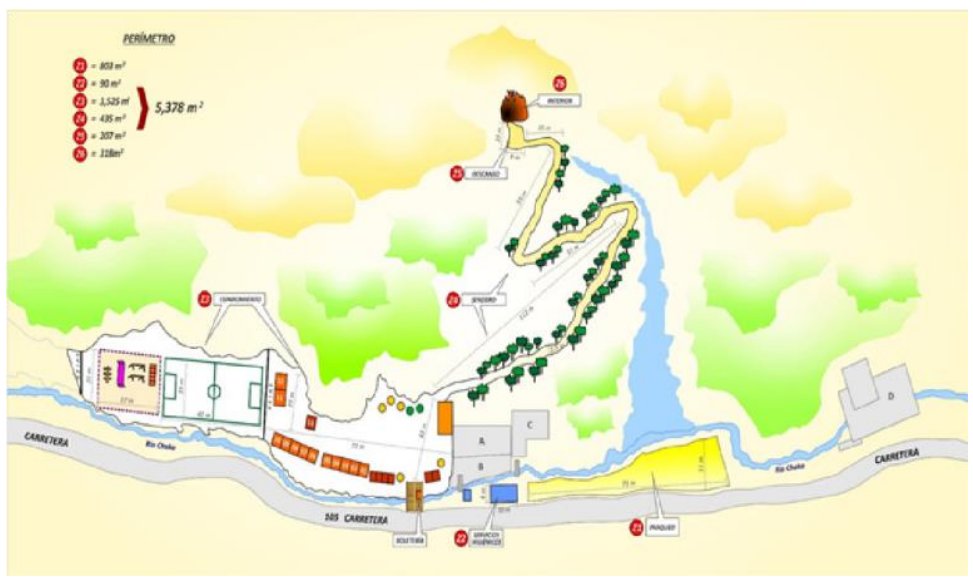

Nota: Z1: parqueo, Z2: servicios higiénicos, Z3: área de esparcimiento, Z4: sendero exterior (desde boletería hasta el ingreso a la gruta), Z5: área de descanso, Z6: sendero interior de la gruta.

\section{CONCLUSIONES}

Las mediciones en campo establecieron una longitud de $525 \mathrm{~m}$. de largo y de ancho en medidas variada, resultando en una superficie de $5378 \mathrm{~m}^{2}$ en la gruta de Huagapo (en sus diferentes zonas). 
Las características que fueron evaluadas evidencian una relación CCF $\geq \mathrm{CCR} \geq \mathrm{CCE}$. Y mencionar que la gestión del atractivo turístico es de manera empírica con índices de eficiencia, según las estadísticas de visitas el último año fue de 49535 (Dircetur - Junin, 2020).

\section{Tabla 6}

Sinopsis de la CCT

\begin{tabular}{|c|c|c|}
\hline \multicolumn{3}{|c|}{ Resumen CCT } \\
\hline \multicolumn{3}{|c|}{ Días laborables para visitar la Gruta de Huagapo: 365 días al año } \\
\hline Descripción & Visitante & \\
\hline & x día & $\mathrm{x}$ año \\
\hline CCF: Capacidad de carga física & 2692 & 982580 \\
\hline Factores de corrección & \multicolumn{2}{|c|}{0.324002} \\
\hline FCsoc: Factor de corrección social & \multicolumn{2}{|c|}{0.547945} \\
\hline FCero: Factor de corrección de erodabilidad & \multicolumn{2}{|c|}{-0.010952} \\
\hline FCacc: Factor de corrección de accesibilidad & \multicolumn{2}{|c|}{0.02229} \\
\hline FCprec: Factor de corrección de precipitación & \multicolumn{2}{|c|}{0.83562} \\
\hline FCbrill: Factor de corrección por brillo solar & \multicolumn{2}{|c|}{0.83993} \\
\hline FCtem: Factor de corrección por cierre temporal & \multicolumn{2}{|c|}{1} \\
\hline FCane: Factor de corrección de anegamiento & \multicolumn{2}{|c|}{0.84248} \\
\hline CCR: Capacidad de carga real & 872 & 318280 \\
\hline CM: Capacidad de manejo & \multicolumn{2}{|c|}{$55.39 \%$} \\
\hline CCE: Capacidad de carga efectiva & 483 & 176295 \\
\hline
\end{tabular}

Las capacidades resultantes como se puede observar en el cuadro son: la capacidad de carga física es de 2692 visitantes al día y al año de 982580 visitantes; la capacidad de carga real, tiene un factor de corrección de 0.324002 considerando los factores de corrección social, erodabilidad, accesibilidad, precipitación, brillo solar, cierre temporal y anegamiento, los cuales ayudaron para el ajuste de la capacidad de carga consiguiendo la cantidad de 872 vistas al día y de 318280 al año como capacidad de carga real ; la capacidad de manejo evaluada previa a una encuesta aplicada a visitantes, ficha de observación de infraestructura, implementación y talento humano, es de 55.39 \% de capacidad de manejo; la capacidad de carga efectiva se consiguió reajustando la capacidad de carga real con la capacidad de manejo y se obtuvo 483 visitantes diarios y de 176295 visitantes al año. Haciendo una comparación de datos relacionados a los visitantes de Gruta de Huagapo según (Dircetur - Junin, 2020) la data histórica de los años fue $(2018=49238$ visitantes nacionales y 297 visitantes internacionales) haciendo un total de 49535 visitantes del año en mención, el cual se considera un $28.09 \%$ del total de la CCE.

El número máximo de visitas CCE no garantiza la sostenibilidad de un atractivo turístico, por el contrario, se debe entender como instrumento que ayuda o promueve la gestión eficiente (Cornejo et al., 2019; Dias et al., 2012). Se establece que la capacidad de carga no debe ser determinante, será una alerta para empoderar, implementar o incrementar talento humano, mejorar la infraestructura y equipamiento, facilitar la 
educación ambiental sostenible, concientización a los visitantes y población circundante con apoyo de los actores directos e indirectos (Cornejo et al., 2019; Caicedo et al., 2018;). La CCT no se debe considerar como un límite estático (Sati, 2018b; Segrado et al., 2017). En la gruta de Huagapo se debe mejorar la CM en relación con infraestructura, instalaciones y talento humano.

\section{AGRADECIMIENTOS}

A la comunidad campesina de Palcamayo y a la junta de administración de la Gruta de Huagapo por brindar facilidades y logística. A la Dirección Regional de Turismo Junín y la Universidad Nacional del Centro del Perú por las fuentes de información secundaria. A la familia Castro Astuvilca por el apoyo a las excursiones e información.

\section{REFERENCIAS BIBLIOGRÁFICAS}

Alvarado, M., Miranda, P., \& Mora, K. (2018). La capacidad de carga turistica como herramienta de planificación en una iniciativa de turismo rural comunitario: Caso de La Posada

Rural La Amistad, Isla de Chira, Golfo de Nicoya Costa Rica. Documenti Geografici, 0(2), 121-143. https://doi.org/10.19246/DOCUGEO2281-7549/201702_07

Amador, E., Cayot, L., Cifuentes, M., Cruz, E., \& Cruz, F. (1996). Determinación de la Capacidad de Carga Turística en los sitios de visita del Parque Nacional de Galápagos (Centro de Patrimonio Mundial de la UNESCO). http://files.admonturistica.webnode.com.co/200000067-81c3882bf6/Capacidad $\% 20 \mathrm{de} \% 20$ carga.pdf

Blancas, F., Gonzales, M., Guerrero, F. M., \& Lozano, M. (2010). Indicadores sintéticos de turismo sostenible: Una aplicación para los destinos turísticos de Andalucía. Revista Electrónica de Comunicaciones y Trabajos de ASEPUMA, 11, 85-118.

Caicedo, D., Benavides, H., \& Carvajal, L. A. (2018). Determinación de la capacidad de carga turística del sendero “Guananguicho”, en la parroquia Huaca, Ecuador. Tierra Infinita, 4(1), 5-19. https://doi.org/10.32645/26028131.741

Cardoso, C. (2006). Turismo Sostenible: Una revisión conceptual aplicada. EL Periplo Sustentable, 11, 5-21.

Castro, R. (2000). Grutas del distrito de Palcamayo: Huagapo, Racasmarca y zonas turísticas.

Cifuentes, M. (1999). Capacidad de carga turística de las áreas de uso público del Monumento Nacional Guayabo, Costa Rica. WWF Centroamérica. http://awsassets.panda.org/downloads/wwfca_guayabo.pdf

Consejo Global de Turismo Sostenible (GSTC), \& Instituto de Turismo Responsable (RTI). (2015). Carta Mundial de Turismo Sostenible ST+20. Cumbre Mundial de Turismo sostenible, Vitoria-Gasteiz, País Vasco, España. http:/ / cartamundialdeturismosostenible2015.com/wp-content/uploads/2016/05 /Carta-Mundial-de-Turismo-Sostenible-20.pdf 
Cornejo, J. L., Chávez, R. M., \& Bravo, M. L. (2019). Capacidad de carga turistica de la Playa Punta Perula y Playa Isla Cocinas como estrategia para un uso turistico sustentable. CIMEXUS, 14(2), 11-26.

Crispin, D., Berovides, V., Marín, J., Garcia, F., \& Fernández- Truan, J. (2017). Límites en la capacidad de carga de visitantes de ecoturismo en Punta del Este, Isla de la Juventud (Cuba). Revista Investigaciones Turísticas, 13, 96-113. https://doi.org/10.14198/INTURI2017.13.05

Del Valle, S. (2011). Uso de indicadores de sostenibilidad en Venezuela. Consideración para el estudio de la sostenibilidad turística. Ecodiseño \& Sostenibilidad, 1(3), 17 33.

Dias, I., Körössy, N., \& Selva, V. F. (2012). Determinación de la capacidad de carga turística: El caso de Playa de Tamandaré - Pernambuco - Brasil. Estudios y perspectivas en turismo, 21(6), 1630-1645.

Dircetur - Junin. (2020). Plan Estratégico Regional de Turismo— PERTUR Junín 2020_ 2025.

El Comercio. (2007). Maravillas del Perú (Empresa Editorial El Comercio S.A.).

Espinoza, N., Fernández, A., Goytia, A., \& Abad, M. (2017, noviembre 22). Local tourism destination carrying capacity measurement challenges. MOVE 2017 5th International Conference on Sub-National Measurement and Economic Analysis of Tourism, Pamplona, Navarre, Spain. http://move2017. inroutenetwork.org/wpcontent/uploads/2017/11/ MOVE2017_proceedings_INTERIM.pdf

Fernández, M., \& Lazovski, O. (2018). La capacidad de carga de un municipio turístico a través de la estimación del tamaño "real" de la población: Dificultades y propuestas. International Journal of Scientific Management and Tourism, 4(3), 93-108.

García, M. (2003). Turismo y conjuntos monumentales. Capacidad de acogida turística y gestión de flujos de visitantes. Tirant lo Blanch. https:/ / editorial.tirant.com/es/libro/turismo-y-conjuntos-monumentales--capac idad-de-acogida-turistica-y-gestion-de-flujos-de-visitantes-maria-garcia-hernandez9788484427575

García, M., De la Calle, M., \& Mínguez, M. del C. (2011). Capacidad de carga turística y espacios patrimoniales. Aproximación a la estimación de la capacidad de carga del conjunto arqueológico de Carmona (Sevilla, España). Boletín de la Asociación de Geógrafos Españoles, 57, 219-241.

Gutierrez-Fernández, F., \& Sierra, S. A. (2015). Cálculo de la capacidad de carga turistica del lago Tarapoto - Puerto Nariño (Amazonas- Colombia). Revista de Tecnología, 14(1), 85-96.

Guzmán-López, T., Borges, O., \& Castillo, A. (2011). Desarrollo económico local y turismo comunitario en países en vías de desarrollo. Un estudio de caso. Revista de CienciasSociales, 12(3), 432-446.

Huari, N., \& Salazar, I. (2006). Palcamayo. Muy cerca de ti. 
Hui, C. (2006). Carrying capacity, population equilibrium, and environment's maximal load. Ecological Modelling, 192(1), 317-320. https://doi.org/10.1016/j.ecolmo del.2005.07.001

Ibañez, R. (2016). Capacidad de carga turística como base para el manejo sustentable de actividades ecoturísticas en Unidades de Manejo Ambiental (UMA) de Baja California Sur (BCS)". El periplo sustentable, 30, 37-76.

Laurente, L., \& Machaca, R. (2020). Modelamiento y proyección de la demanda de turismo internacional en Puno-Perú. Revista Brasileira de Pesquisa em Turismo, 14(1), $34-$ 55. https://doi.org/10.7784/rbtur.v14i1.1606

López, J. M., \& López, L. M. (2008). La capacidad de carga turística: Revisión crítica de un instrumento de medida de sostenibilidad. El Periplo Sustentable, 15, 123-150. https://doi.org/10.21854/eps.v0i15.938

Marozzi, P., Lima, J., \& Sarmiento, M. (2011). Caracterización de los Visitantes y Determinación de la Capacidad de Carga Turística en el Parque Nacional Los Cardones (PNLC). Salta, Argentina. Ciencia, 6(22), 7-25.

Matos, L., \& Pérez, S. (2019). Revisión sobre capacidad de carga turística y la prevención de problemas ambientales en destinos emergentes. Turismo y Sociedad, 24, 77-100.

Mc Coy, C. (2016). Evaluación De La Capacidad De Carga Turística Como Elemento De Análisis Del Desempeño De

Un Destino Turístico: Caso Cancún. Revista Internacional Administración \& Finanzas, $9(6), 59-80$.

Mestanza, C., Llanos, D., \& Herrera, R. (2019). Capacidad de carga turística para el desarrollo sostenible en senderos de uso público: Un caso especial en la reserva de producción de fauna Cuyabeno, Ecuador. Caribeña de Ciencias Sociales, marzo. https://www.eumed.net/rev/caribe/2019/03/reserva-producccion-fauna.html

Montalvo, R., \& Castillo, J. (2018). Estimación de la capacidad de carga Turística en Agua Selva (Tabasco - México). Base para la planificación y el desarrollo regional. 22.

Organización de la Naciones Unidas (ONU) (Ed.). (1992). Declaración de Río sobre el Medio Ambiente y el Desarrollo. http://portal.uned.es/pls/portal/docs/ PAGE/UNED_MAIN/LAUNIVERSIDAD/DEPARTAMENTOS/0614/ASI GNAT/MEDIOAMBIENTE/TEMA \%201/\%20\%20\%20\%20\%20DECLARA CI\%C3\%93N\%20DE\%20R\%C3\%8DO\%201992.PDF

Organización de las Naciones Unidas (ONU). (2012). El futuro que queremos. 59. https://rio20.un.org/sites/rio20.un.org/ files/a-conf.216-1-1_spanish.pdf.pdf

Organización de las Naciones Unidas (ONU), Organización Mundial del Turismo (OMT), \& Unión Europea (UE) (Eds.). (1995). Carta del Turismo sostenible. http://www.datosdelanzarote.com/uploads/doc/20051226123220895CartaTuris moLanzarote.pdf

Perruolo, G., \& Camargo, C. (2017). Estimación de capacidad de carga turística en el área Chorro El Indio, estado Táchira, Venezuela. Cuadernos de Geografía: Revista 
Colombiana de Geografía, 26(2), 77-90. https://doi.org/10.15446/rcdg. v26n2.59259

Puente, E. D., Pérez, C. A., \& Solís, C. I. (2011). Capacidad de carga en senderos turísticos del Centro de Cultura para la conservación para la conservación Piedra Herrada, México. Revista de Estudios Territoriales, 13(2), 93-114.

Rosell, M. P., \& Del Pozo, O. (2007). Reserva Natural Isla de Puan. Propuestas metodológicas de conservación. Aportes y Transferencias, 1(11), 61-84.

Salerno, F., Viviano, G., Manfredi, E. C., Caroli, P., Thakuri, S., \& Tartari, G. (2013). Multiple Carrying Capacities from a management-oriented perspective to operationalize sustainable tourism in protected areas. Journal of Environmental Management, 128, 116-125. https://doi.org/10.1016/j.jenvman.2013.04.043

Sati, V. P. (2018a). Carrying capacity analysis and destination development: A case study of Gangotri tourists/pilgrims' circuit in the Himalaya. Asia Pacific Journal of Tourism Research, 23(3), 312-322. https://doi.org/10.1080/10941665.2018.143 3220

Sati, V. P. (2018b). Carrying capacity analysis and destination development: A case study of Gangotri tourists/pilgrims' circuit in the Himalaya. Asia Pacific Journal of Tourism Research, 23(3), 312-322. https://doi.org/10.1080/10941665.2018.14 33220

Saveriades, A. (2000). Establishing the social tourism carrying capacity for the tourist resorts of the east coast of the Republic of Cyprus. Tourism Management, 21(2), 147-156. https://doi.org/10.1016/S0261-5177(99)00044-8

Segrado, R., González, C. A., Arroyo, L., \& Quiroga, B. (2017). Capacidad de carga turística y aprovechamiento sustentable de Áreas Naturales Protegidas. CIENCIA ergo-sum, 24(2), 164-172.

Simancas, M. R. (2006). Los modelos de uso turístico de las áreas protegidas de Canarias: Una propuesta metodológica. Investigaciones Geográficas, 39, 25-45. https://doi.org/10.14198/INGEO2006.39.02

Soria-Díaz, H. F., \& Soria-Solano, B. (2015). Determinación de la capacidad de carga turística en los sitios de visita de la Reserva Nacional Allpahuayo-Mishana, Loreto, Perú. Ciencia Amazónica (Iquitos), 5(1), 25-34. https://doi.org/10.22386/ca. v5i1.87

Tudela, L., \& Giménez, A. (2008). Determinación de la capacidad de Carga Turística de la capacidad de carga turística en tres senderos de pequeño recorrido en el Municipio de Cehegín (Murcia). Cuadernos de Turismo, 22, 211-229.

Varisto, Y., Rosell, P., \& Rosake, P. (2009). Capacidad de carga turística en área de humedales. Aportes y Transferencias, 2(13), 44-64.

Vera, J. F., Palomeque, F. L., Marchena, M., \& Antón, S. (2011). Análisis territorial del turismo y planificación de destinos turísticos. Tirant lo Blanch. https:/ / dialnet.unirioja.es/servlet/libro?codigo $=675851$ 
Wearing, S., \& Neil, J. (1999). Ecoturismo: Impacto, Tendencias y posibilidades. Editorial Síntesis. https://www.casadellibro. com/libro-ecoturismo-impacto-tendencias-yposibilidades/9788477387824/727101

World Tourism Organization (UNWTO) (Ed.). (2018). UNWTO Tourism Highlights: 2018 Edition. World Tourism Organization (UNWTO). https://doi.org/ $10.18111 / 9789284419876$

World Tourism Organization (UNWTO) (Ed.). (2019). International Tourism Highlights, 2019 Edition. World Tourism Organization (UNWTO). https://doi.org/10. 18111/9789284421152

Yang, H. (2017). Study on the optimization model of tourism environmental carrying capacity based on tourism planning. Revista de La Facultad de Ingeniería, 32(12), 1082-1089.

Zhang, Y., Li, X., \& Su, Q. (2017). Does spatial layout matter to theme park tourism carrying capacity? Tourism Management, 61, 82-95. https://doi.org/10.1016/j. tourman.2017.01.020

\section{CITAR COMO:}

Huaroc Ponce, E. J., Raqui Ramirez, C. E., Jurado Taipe, R. S., \& Huaroc Ponce, N. M. (2021). Capacidad de carga Turística, base para el manejo sustentable: Gruta de Huagapo. Puriq, 3(1), 39-54. https://doi.org/10.37073/puriq.3.1.114 